\section{Autosomal-rezessive Vererbung}

\section{J. Arnemann}

Abteilung Molekulargenetik, Labor Dr. Wisplinghoff, Köln, Deutschland

\section{Synonym(e) AR}

Englischer Begriff Autosomal-recessive inheritance

Definition Bei autosomal-rezessiver Vererbung tritt eine einzelne sog. rezessive Kopie (heterozygot) phänotypisch nicht in Erscheinung, sondern nur in doppelter Kopienzahl (homozygot).

Beschreibung Eine autosomal-rezessive Vererbung beschreibt 2 wesentliche Eigenschaften, nämlich eine Zuordnung der Eigenschaft oder der Krankheitsursache auf ein Autosom, d. h. einem Nicht-Geschlechtschromosom mit gleichzeitigem Ausschluss einer X-chromosomalen Vererbung, sowie die Tatsache, dass diese entsprechende Eigenschaft oder auch Krank- heitsmutation in der Expression schwächer ist als andere und diesen im heterozygoten Zustand in der phänotypischen Ausprägung unterlegen ist. Die rezessiven, unterdrückten Eigenschaften werden nur im homozygoten Zustand, d. h. bei Vorhandensein von 2 Kopien phänotypisch ausgeprägt. Formalgenetisch hat ein Ehepaar, bei dem beide Partner heterozygot für eine autosomal-rezessive Eigenschaft oder Mutation sind, mit einer Wahrscheinlichkeit von $25 \%$ ein Kind, das diese rezessive Eigenschaft im homozygoten Zustand ausbildet. Die häufigsten autosomal-rezessiven Erkrankungen in der Praxis sind Mukoviszidose und - vor allem in der mediterranen Bevölkerung - das familiäre Mittelmeerfieber.

Der Begriff einer autosomal-rezessiven Vererbung ist von Bedeutung in der genetischen Beratung und der formalen genetischen Beschreibung eines Vererbungsmechanismus.

\section{Literatur}

Strachan T, Read AP (2005) Molekulare Humangenetik. Elsevier $\mathrm{GmbH}$, München 